\title{
Birthweight Discordance in Multiple Pregnancy
}

\author{
Isaac Blickstein' and Robin B. Kalish ${ }^{2}$ \\ ' Dept. of Obstetrics and Gynecology, Kaplan Medical Center, Rehovot, and the Hadassah-Hebrew University School of Medicine, Jerusalem, Israel \\ 2 Division of Maternal Fetal Medicine, Department of Obstetrics and Gynecology, Weill Medical College of Cornell University, New York, NY
}

\begin{abstract}
This paper reviews several aspects of discordant growth in multiple pregnancies. Discordant growth is not a chance event and therefore several patterns can be discerned. About $75 \%$ of twins exhibit $<15 \%$ discordance (concordant), $20 \%$ are $15-25 \%$ (mildly) discordant, and about $5 \%$ are more than $25 \%$ (severely) discordant. Higher frequencies and increased severity are seen among triplets. Five observations regarding discordance became generally accepted: (a) not all discordant pairs are similar; (b) the larger the discordance level the greater is the risk for an adverse outcome; (c) discordant growth does not necessarily represent growth restriction; (d) a discordance level may have a different clinical implication in different gestational ages; and (e) the smaller fetuses in severely discordant pairs are at disproportionate risk for neonatal mortality. Mild discordance may represent a normal variation between sibs whereas severely discordant pairs often exhibit patterns of growth restriction. Not infrequently, discordance may represent an adaptation to the limited intrauterine space in order to increase gestational age.
\end{abstract}

\section{The Definition}

Birthweight discordance occurs whenever there is disparity in birthweights between the larger and the smaller infant of a multiple pregnancy set. This difference, however, might be very small or relatively large. When one analyzes a large series of multiples, one seldom finds that all members of the set have the same birthweight. Indeed, some variation is expected between sibs and therefore the magnitude of the difference - the degree of discordance - has to be incorporated in the definition.

Three definitions of discordant birthweights have been used routinely (Blickstein \& Lancet, 1988). The first the so-called "absolute" definition records the absolute birthweight difference. This definition, however, assigns the same degree of discordance (i.e., $500 \mathrm{~g}$ ) to a twin pair weighing $1500 / 1000 \mathrm{~g}$ and to a pair weighing $3000 / 2500 \mathrm{~g}$. The second definition is the so-called "percent" definition, whereby the birthweight disparity is calculated as a percentage of the larger infant. The percent definition is the by far the most common in practice. However, as is the case with the absolute definition, it also does not refer to the actual size of the sibs. Thus, the percent definition may assign the same degree of discordance (i.e., 20\%) to a twin pair weighing 1500/1200g and to a pair weighing 3000/2400g. The third definition, the so-called "statistically derived" definition, refers to the extreme of the distribution of discordance values (presented by the "percent" definition), such as the 95th percentile or one or two standard deviations above the mean. Despite its potential statistical relevance, one needs a large sample size - preferably population-based - to derive these values. Moreover, we have recently shown in a large series of 134,385 twin pairs that discordance does not have a normal (Gaussian) distribution and central measures like the mean $\pm S D$ do not accurately describe the situation at large or by gestational age (Kalish et al., 2002). It is therefore advisable that if a "statistically derived" definition is favored, one should use only the "percentile" definition to define the degree of discordance by statistical methods (Redman et al., 2002).

The definition of birthweight discordance is even more complex in triplets. Authors usually borrowed the "percent" definition of twins and used the difference between the largest and smallest triplet of each set (Fountain et al., 1995; Jones et al., 1991; Mordel et al., 1993). However, this definition ignores the middle one, and as a result, the true inter-triplet relationship. We therefore proposed a new description in which the relative birthweight of the middle triplet was defined (Blickstein et al., 2003a). We initially used the percent definition to define concordance and discordance. The middle triplet was defined as symmetrical when its birthweight was within $\pm 25 \%$ of the average birthweight between the larger and smaller triplet, as lowskew when a set comprised one large and two small triplets, and defined as high-skew if the set comprised two large and one small triplets. Such designations may denote different severity, but interestingly, the frequencies of different types of triplet discordance did not change with gestational age, suggesting three distinct, gestational-age independent, types of discordant growth in triplets (average values: symmetrical $57 \%$, high-skew $30 \%$, and low-skew $13 \%$ ). The data further indicate that symmetrically discordant sets are probably the standard arrangement favored by the uterine environment and among discordant sets, one rather than two of the triplets was more often smaller.

\section{Incidence}

When large samples sizes are evaluated, it could be shown that the frequency of discordant pairs decreases as a polynomial function with increasing discordance levels.

Address for correspondence: Isaac Blickstein, MD, Dept. of Obstetrics and Gynecology, Kaplan Medical Center, 76100 Rehovot, Israel.Email: blick@netvision.net.il 
The frequency drops from around 30\% at discordance level of $<5 \%$ to less than $4 \%$ for discordance of $25-30 \%$ and further to less than $1 \%$ for discordance of more than $40 \%$ (Kalish et al., 2002). The cumulative frequency curve in Figure 1, shows that about $75 \%$ of twins exhibit $<15 \%$ discordance, an additional 20\% are $15-25 \%$ discordant, and about $5 \%$ are more than $25 \%$ discordant. These levels of discordance have been referred to as concordant, mild discordant, and severe discordant levels (Blickstein \& Lancet, 1988).

We have recently compared the percent definition (> 25\% discordance) with the 95th percentile of discordance by gestational age (Kalish et al., 2002). It could be shown that in terms of neonatal mortality, both definitions can be used interchangeably. However, it is important to remember that we examined only neonatal mortality, and information about maternal or neonatal morbidity was not included in the analysis.

An important related issue is the birth order of the smaller twin in a discordant set. Many series comparing small/large versus large/small sets confirmed the logical assumption that if discordance were a chance event, either twin would have the same chance of being the smaller one. However, at the same time, it was a common belief that the smaller twin is usually the second-born. The controversy can be settled if one adds the inter-twin discordance level to the equation. At lower levels of discordance either twin can be the smaller but the likelihood of the second-born twin to be the smaller increases with increasing discordance levels (Blickstein et al., 1987; Blickstein et al., 1999). At discordance levels $>25 \%$, the smaller twin was 3 to 6 times more often the second-born (Blickstein et al., 1999).

Observations in triplets suggest a higher frequency and severity of birthweight discordance in triplets. Using the percent definition, Jones et al. (1991) found that $30.4 \%$ of 196 sets exhibited at least $25 \%$ discordance, a figure similar to the nearly $25 \%$ found by Mordel et al. (1993) and $34.2 \%$ found by Fountain et al. (1995). Extreme discordance over $40 \%$ was found in 7 (Jones et al., 1991) to $11.8 \%$ (Fountain et al., 1995) of the cases. These percentages are much larger than those reported in twins. Using a much larger database than in previous reports, we found discordance of $25.1-35 \%$ and $>35 \%$ in $19.4 \%$ and $9.5 \%$ of the 2804 triplets analyzed, respectively, thrice higher than the $3.1 \%$ found in twins (Blickstein et al., 2003a).

Any discussion on the incidence of discordance should acknowledge the fact that all cited frequencies do not represent the natural frequencies but may be a direct result of iatrogenic preterm deliveries indicated (wrongly or not) for growth discordance. In fact, the true incidence of discordance by gestational age might represent better the effect of the natural growth pattern of twins modulated by the policy of intervention.

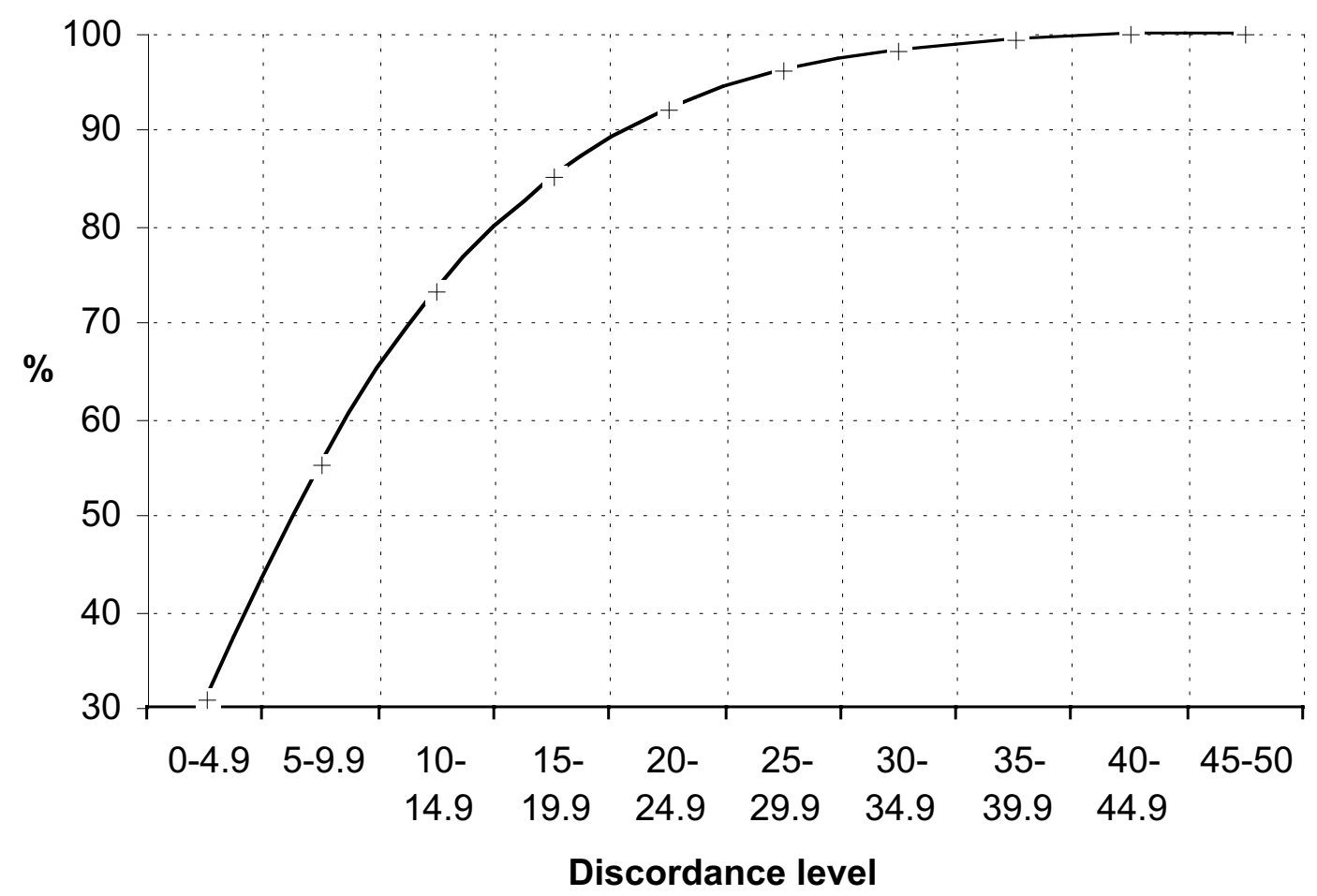

\section{Figure 1}

Cumulative frequencies by discordance level.

Note: Data derived from 134,385 twin pairs, United States 1995-1997 Multiple Birth Matched Data Set. Kalish RB, Branum A, Keith LG, Sharma G, Blickstein I (unpublished observation). 


\section{Is Birthweight Discordance a Normal Variation?}

In twins, especially twins of different sex, one may expect some degree of discordance to represent a normal variation between sibs. The magnitude of this variation, in terms of discordant birthweight, is still controversial and the controversy is further confounded by conflicting outcomes of discordant twins. At the same time, five observations became generally accepted: (a) not all discordant pairs are similar and some are doing better than others; (b) the larger the discordance level the greater is the risk for an adverse outcome (Blickstein et al., 2000; Cheung et al., 1995; Demissie et al., 2002; Hollier et al., 1999); (c) discordant growth (relative growth restriction) does not necessarily represent absolute growth restriction; (d) the same discordance level may have a different clinical connotation in different gestational ages (Cheung et al., 1995); and (e) pairs affected by discordance of $25 \%$ or more are at disproportionate risk for neonatal mortality when compared with concordant smaller or larger twins (Branum \& Schoendorf, 2003).

To avoid the potentially confounding effect of gestational age, we proposed a model whereby the total twin birthweight is assumed to represent the capacity of the uterine milieu to nurture twins at any given time. If this were the case, one would expect comparable frequencies of discordance across gestational ages and total twin birthweights. However, when the frequencies of birthweight discordance of $>25 \%$ were counted across the deciles of the total twin birthweight, the frequencies decreased in a nonlinear, inversely logarithmic, trend (Blickstein et al., 1999). This observation suggested that the more favorable the uterine milieu (i.e., larger total birthweight deciles) for carrying twins the smaller the likelihood of discordant twin growth. This conclusion was supported by showing that at discordance level of $15-24.9 \%$, the frequency function was inversely linear, as would have been expected with a normal variation (Blickstein et al., 2000). However, at levels $25-34.9 \%$ and over $34.9 \%$ discordance, the observed patterns of birthweight discordance did not substantiate a normal variation, as both frequency functions were inversely logarithmic with a much steeper decrease at the $\geq 35 \%$ level (Blickstein et al., 2000).

\section{Discordance as an Adaptive Measure}

The data from the above-mentioned studies (Blickstein et al., 1999; Blickstein et al., 2000) suggest that there are three levels of discordance. In the lowest levels (probably at $<25 \%$ ), discordance seems to be related to the normal variation expected from the natural dissimilarities between sibs. In the highest level (probably > 35\%), discordance seems to be related to the exhausted uterine environment and reflects growth restriction. The clinical approach to both levels is generally accepted: observation or intervention for the lowest and highest degrees of discordance, respectively. However, between these levels are twins whose different birthweights neither are not a result of a normal variation nor are they representing genuine growth restriction. This group comprises $10 \%$ of the entire twin population and is of special interest because of the controversies involved in their clinical management.
One way to explain the relative growth restriction within the framework of normal outcome was to consider growth discordance as an adaptive measure to promote maturity. This hypothesis suggests that within a limited uterine environment, the combination of one larger and one smaller twin may reduce uterine over-distension and increase gestational age.

Several studies indirectly support this assumption. The first was to determine if discordant twins are indeed delivered at a more advanced gestational age than concordant twins of the same total twin birthweight (Blickstein \& Goldman, 2003). Using the United States National Center for Health Statistics matched multiple birth data file, we compared the mean gestational age between concordant and discordant $(>25 \%)$ pairs at $250 \mathrm{~g}$ total birthweight intervals. The mean gestational age of discordant pairs was significantly higher across the entire range of total birthweight intervals except for the upper interval (> 4750g). The same study was recently repeated by using the Swedish twin database, yielding the same results (Hirsh, 2003).

Another indirect support for the hypothesis comes from the important effect of parity on fetal growth in multiples. Indeed, the effect of discordance on gestational age was modified by parity, with significant differences between concordant and discordant pairs among primiparas mainly at the lower birthweight strata (Blickstein \& Goldman, 2003). In triplets, the parity effect was similar and primiparas had significantly fewer concordant sets as a result of fewer moderately discordant sets and more symmetrically discordant sets (Blickstein et al., 2003a). Further support to the notion that uterine volume affects the growth of multiples comes from large cohort of United States live-born triplets, showing a significant positive correlation between the mean total triplet birthweight and maternal height (Blickstein et al., 2003b).

Finally, it has been recently shown that even among severely discordant twins there is a substantial group in which there is no genuine growth restriction. (Blickstein \& Keith, in press). We studied 10,683 pairs exhibiting > 25\% discordance. The pairs were classified according to the birthweight of the smaller twin, being $<10$ th, 10th to 50 th, or $>50^{\text {th }}$ percentile. It has been found that only about $60 \%$ of severe discordance is indeed associated with an SGA twin. Consequently, neonatal mortality rate was significantly higher among pairs in which the smaller twin weighed $<10$ th birthweight percentile, a difference that results from the higher mortality among the smaller but not among the larger twins (Blickstein \& Keith, in press; Branum \& Schoendorf, 2003).

The obvious dissimilarity between discordance in twins and triplets, in terms of frequencies and severity, suggests a different, plurality-related, conceptual approach to birthweight discordance. For instance, the polynomial function describing the frequencies of $>25 \%$ discordant triplet sets and total birthweight deciles (Blickstein et al., 2003a) is different from the inverse logarithmic relationship found in twins (Blickstein et al., 1999). From a clinical perspective, the difference between the two functions seems to be consistent with the difference between the uterine capacities: whereas in twins "capacity" is associated with an inversely 
logarithmic decline of the frequencies of discordant pairs, the uterine environment in triplets is unable to limit the frequencies of discordant sets as the uterine size increases. The general observation, however, remains the same for twins and triplets: the more favorable the uterine milieu the smaller the likelihood of discordant growth (Blickstein et al., 2002).

\section{Mechanisms of Discordance}

The mechanisms which underlie the discordance phenomenon are not clear. From the analysis of growth curves of twins and triplets, it is however clear that at the same time the average individual twin or a triplet is smaller than the average matched for gestational age singleton, relatively few multiples have birthweights compatible with the definition of SGA for singletons (Blickstein, 2002). Moreover, the entire fetal "mass" of a multiple pregnancy is, in fact, growth promoted compared to that of singletons, as can be appreciated from the absolute gain in fetal "mass" in twin and triplet weight over that of the 90th birthweight percentile of singletons (Blickstein, 2002).

It can be shown that the utero-placental unit is still very efficient also in cases of discordant twins. Figure 2 is a plot of the "excess fetal mass" in > 25\% discordant twins ( $n=12,846)$ compared to the median birthweight of singletons $(n=2,966,882)$ (USA National Center for Health Statistics). Figure 2 shows that in early discordance $(<28$ weeks), the utero-placental unit produces more than $75 \%$ "fetal mass" compared to singletons. The excess mass is gradually decreased to around 50\% "excess fetal mass", a value that remains quite unchanged with advanced pregnancy. These data suggest that even when severe discordance occurs in twin pregnancies, the utero-placental unit still provides 50 to $75 \%$ more than for the average singleton gestation. It could be also suggested that whatever mechanism is involved in the discordance phenomenon, it is less likely to be related to a generalized uterine factor and it is more likely to be related to the individual twin or triplet fetus and its respective placental mass. A study of triplets (Blickstein et al., 2002) clearly suggests that each triplet fetus has its own linear growth pattern, with a significantly different inclination than that of its sibs. The resultant discordant growth in triplets is assumed to be a physiological, differential, and gestational age-dependent phenomenon (Blickstein et al., 2002).

Several attempts to evaluate the placental origin of discordance have been made, mainly in correlating placental structure (i.e., velamentous cord insertion), chorionicity (unequal placental sharing), size, and pathological lesions. Bleker et al. (1988) studied placental indices (placental weights related to birthweights) by plurality. Although the placental index was similar in singletons, twins and triplets, it was smaller in multiples delivered after 24 weeks. The term "placental crowding" was used to denote poor early placental development preceding growth restriction in multiples. Victoria et al. (2001) correlated pregnancy outcomes and placental findings among severely discordant twins. Placentas of the smaller fetus in dichorionic twins with separate placentas and the entire placenta in monochorionic twins were significantly lighter in severely discordant twins than in concordant and mildly discordant controls. In contrast, Eberle and co-workers (1993) found that birthweight discordance $>20 \%$ was not attributable to differences in placental weight but to a significantly greater number of placental lesions in the lighter twin than in the heavier twin. These opposing views cannot be settled because it is

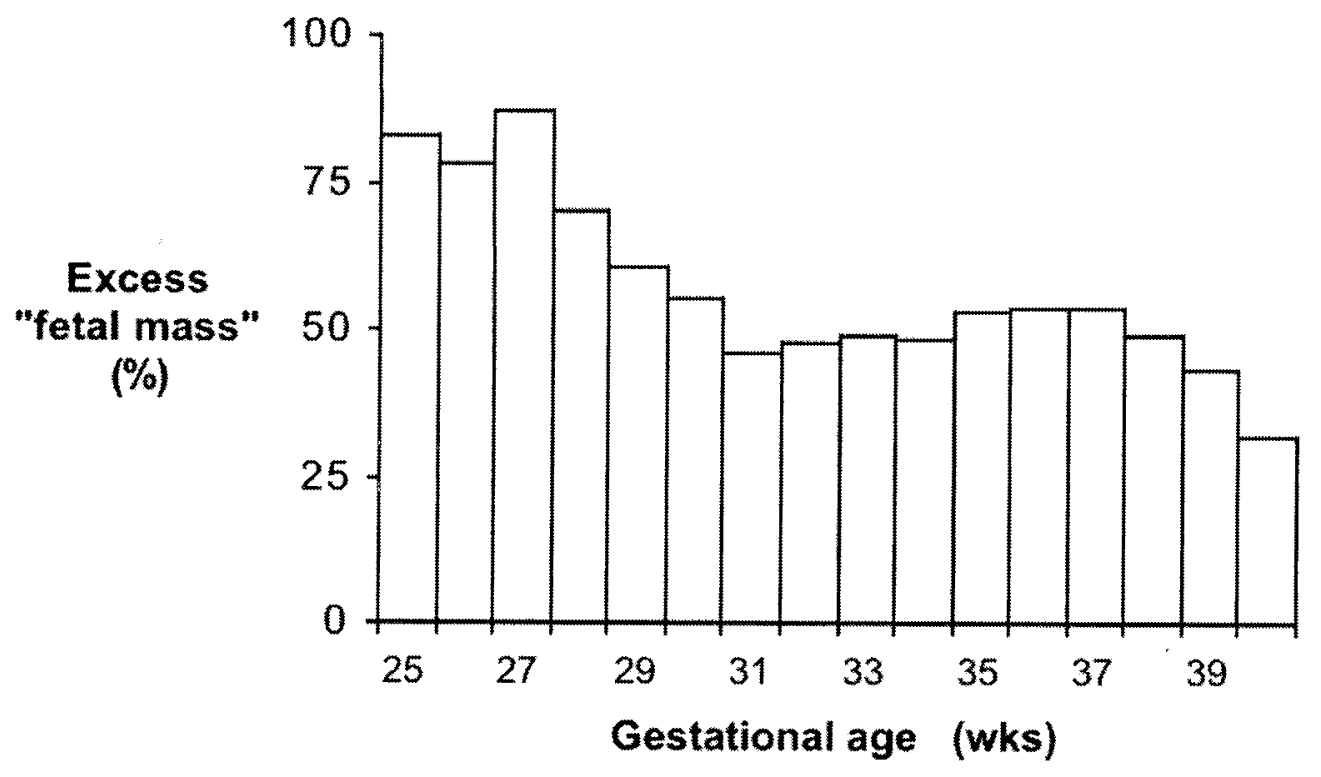

Figure 2

Excess of fetal mass in $>25 \%$ discordant twins $(n=12,846)$, expressed as percentage of the median singleton birthweight $(n=2,966,882)$.

Note: Based on data source from the USA National Center for Health Statistics. 
currently impossible to determine if the diminished rate of placental growth lags behind, parallels, or exceeds the rate of diminished fetal growth.

Finally, correlation between placental function and discordance has been attempted by measuring maternal and fetal concentrations of insulin-like growth factor-I (IGF-I), IGF-II and insulin-like growth factor binding protein-1 in twins with or without discordant birthweight (Westwood et al., 2001). The data indicate that growth discordance of twins exposed to the same maternal environment may be due to variations in either of these placenta-function depending factors (Westwood et al., 2001).

\section{Fetal Gender and Discordance}

Males weigh more than females, a difference attributed to genotypic and phenotypic gender differences (Blumrosen et al., 2002). If differences in birthweights between males and females were just "structural", one should expect the mean birthweight of unlike-sex pairs to be similar to the average between female-female and male-male pairs, but not skewed towards either. However, it has been repeatedly shown that the presence of a male fetus improves the uterine growth environment, and females in unlike-sex pairs tend to have higher birthweights compared with females in like-sexed DZ pairs (Glinianaia et al., 1998). In the subset of discordant pairs, the frequency of females among smaller twins was significantly higher than that of males, implying that males are doing better in the uterine environment of a twin gestation (Blickstein \& Weissman, 1990). This conclusion was indirectly supported by a study showing that the mean birthweight of males in unlike-sex pairs was significantly higher than that of males in malemale pairs, with no such relation seen in females (Loos et al., 2001). Blumrosen et al. (2002) showed significantly higher birthweights of females in unlike-sex pairs, a trend existing irrespective of discordance level. The implications of gender-dependent divergent birthweight might be more complex when one may speculate that female fetuses growing in an androgenic-anabolic environment may subsequently exhibit some form of hormone-mediated manifestation during adulthood.

\section{References}

Bleker, O. P., Oosting, J., \& Hemrika, D. J. (1988). On the cause of the retardation of fetal growth in multiple gestations. Acta Geneticae Medicae et Gemellolgica, 37, 41-46.

Blickstein, I., Shoham-Schwartz, Z., Lancet, M., \& Borenstein, R. (1987). Characterization of the growth-discordant twin. Obstetrics and Gynecology, 70, 11-15.

Blickstein, I., \& Lancet, M. (1988). The growth discordant twin. Obstetrical and Gynecological Survey, 43, 509-515.

Blickstein, I., \& Weissman, A. (1990). Birthweight discordancy in male-first and female-first pairs of unlike-sexed twins. American Journal of Obstetrics and Gynecology, 162, 661-663.

Blickstein, I., Goldman, R. D., Smith-Levitin, M., Greenberg, M., Sherman, D., \& Rydhstroem, H. (1999). The relation between inter-twin birthweight discordance and total twin birthweight. Obstetrics and Gynecology, 93, 113-116.
Blickstein, I., Goldman, R. D., \& Mazkereth, R. (2000). Adaptive growth restriction as a pattern of birthweight discordance in twin gestations. Obstetrics and Gynecology, 96, 986-990.

Blickstein, I., Jacques, D. L., \& Keith, L. G. (2002). Total and individual triplet birth weigh as a function of gestational age. American Journal of Obstetrics and Gynecology, 186, 1372-1375.

Blickstein, I. (2002). Normal and abnormal growth of multiples. Seminars in Neonatology, 7, 177-185.

Blickstein, I., \& Goldman, R. D. (2003). Inter-twin birthweight discordance as a potential adaptive measure to promote gestational age. Journal of Reproductive Medicine, 48, 449-454.

Blickstein, I., Jacques, D. L., \& Keith, L. G. (2003a). A novel approach to intertriplet birthweight discordance. American Journal of Obstetrics and Gynecology, 188, 172-176.

Blickstein, I., Jacques, D. L., \& Keith, L. G. (2003b). The effect of maternal height on gestational age and birthweight in nulliparous mothers of triplets with normal pre-gravid body mass index. Journal of Reproductive Medicine, 48, 335-338.

Blickstein, I., \& Keith, L.G. (in press) Neonatal mortality among growth discordant twins classified according to the birth weight of the smaller twin. American Journal of Obstetrics and Gynecology.

Blumrosen, E., Goldman, R. D., \& Blickstein, I. (2002). Growth discordance and the effect of a male twin on birthweight of its female co-twin: A population-based study. Journal of Perinatal Medicine, 30, 510-513.

Branum, A. M., \& Schoendorf, K. C. (2003). The effect of birthweight discordance on twin neonatal mortality. Obstetrics and Gynecology, 101, 570-574.

Cheung, V. Y., Bocking, A. D., \& Dasilva, O. P. (1995). Preterm discordant twins: What birthweight difference is significant? American Journal of Obstetrics and Gynecology, 172, 955-959.

Demissie, K., Ananth, C. V., Martin, J., Hanley, M. L., MacDorman, M. F., \& Rhoads, G. G. (2002). Fetal and neonatal mortality among twin gestations in the United States: The role of intrapair birthweight discordance. Obstetrics and Gynecology, 100, 474-480.

Eberle, A. M., Levesque, D., Vintzileos, A. M., Egan, J. F., Tsapanos, V., \& Salafia, C. M. (1993). Placental pathology in discordant twins. American Journal of Obstetrics and Gynecology, 169, 931-935.

Fountain, S. A., Morrison, J. J., Smith, S. K., \& Winston, R. M. (1995). Ultrasonographic growth measurements in triplet pregnancies. Journal of Perinatal, 23, 257-263.

Glinianaia, S. V., Magnus, P., Harris, J., \& Tambs, K. (1998). Is there a consequence for fetal growth of having an unlike-sexed cohabitant in utero? International Journal of Epidemiology, 27, 657-659.

Hirsch, N. (2003). Birth weight discordance as an adaptive measure to promote fetal maturity. Unpublished thesis.

Hollier, L. M., McIntire, D. D., \& Leveno, K. J. (1999). Outcome of twin pregnancies according to intrapair birthweight differences. Obstetrics and Gynecology, 94, 1006-1010.

Jones, J. S., Newman, R. B., \& Miller, M. C. (1991). Cross-sectional analysis of triplet birthweight. American Journal of Obstetrics and Gynecology, 164, 135-140.

Kalish, R. B., Barnum, A. M., Keith, L. G., Sharma, G., Blickstein, I. (2002). Unpublished observation. 
Loos, R. J. F., Derom, C., Eackels, R., Derom, R., \& Vlietinck, R. (2001). Length of gestation and birthweight in dizygotic twins. Lancet, 358, 560-561.

Mordel, N., Benshushan, A., Zajicek, G., Laufer, N., Schenker, J. G., \& Sadovsky, E. (1993). Discordancy in triplets. American Journal of Perinatology, 10, 224-225.

Redman, M. E., Blackwell, S. C., Refuerzo, J. S., Kruger, M., Naccasha, N., Hassan, S. S., et al. (2002). The ninety-fifth percentile for growth discordance predicts complications of twin pregnancy. American Journal of Obstetrics and Gynecology, 187, 667-671.
Victoria. A., Mora, G., \& Arias, F. (2001). Perinatal outcome, placental pathology, and severity of discordance in monochorionic and dichorionic twins. Obstetrics and Gynecology, 97, 310-315.

Westwood, M., Gibson, J. M., Sooranna, S. R., Ward, S., Neilson, J. P., \& Bajoria, R. (2001). Genes or placenta as modulator of fetal growth: Evidence from the insulin-like growth factor axis in twins with discordant growth. Molecular and Human Reproduction, 7, 387-395 